\title{
Analysis and synthesis of feedback systems: quadratic functions and LMIs
}

\author{
Luca Zaccarian (with Andrew R. Teel)
}

June 29, 2009

\section{Introduction}

This note was given as a handout at the 2009 Bertinoro summer school on robust control with constraints. The following paper are appended at the end of the file, because they contain further develoopment along the directions given in the note:

- LMI-based anti-windup with exponentially stable plants [12]: it contains the development of static and dynamic anti-windup with global sector characterizations (see also [14] for the external anti-windup case and [9] for the reduced order design);

- LMI-based anti-windup with any plant [17]: it contains the development of static and dynamic antiwindup with regional sector characterizations. A discrete-time version of these results is given in $[22]$.

- Analysis of stability and performance with quadratic, max of quadratics and convex hull of quadratics [18]. This is an extended version of the paper [16].

- Analysis of stability and performance with piecewise quadratic Lyapunov functions [5].

- LMI-based bounded stabilization (not anti-windup!) using controller with internal deadzone loops [4].

Additional references that could give insight into the anti-windup approaches are the survey [25] and the work [10] and references therein, where the Model Recovery Anti-windup (formerly called $\mathcal{L}_{2}$ anti-windup) is used. This solution was not covered in the Bertinoro class.

\subsection{Description of feedback loop}

This chapter starts by addressing analysis of feedback loops with saturation. In particular, it develops tools for verifying internal stability and quantifying $\mathcal{L}_{2}$ external stability for the well-posed feedback interconnection of a linear system with a saturation nonlinearity. Such a system is depicted in Figure 1 and has state-space representation

$$
\widetilde{\mathcal{H}}\left\{\begin{aligned}
\dot{x} & =\widetilde{A} x+\widetilde{B} \sigma+\widetilde{E} w \\
z & =\widetilde{C} x+\widetilde{D} \sigma+\widetilde{F} w \\
u & =\widetilde{K} x+\widetilde{L} \sigma+\widetilde{G} w
\end{aligned}\right.
$$

It is more convenient for analysis and synthesis purposes to express this system in terms of the deadzone nonlinearity $q=u-\operatorname{sat}(u)$ as shown in Figure 2. The state-space representation of (1) using the deadzone nonlinearity is obtained by first solving for $u$ in the equation

$$
u=\widetilde{K} x+\widetilde{L} u-\widetilde{L} q+\widetilde{G} w .
$$

The solution can be obtained when $I-\widetilde{L}$ is invertible, which is a necessary condition for well-posedness of the feedback system (1). For more information on well-posedness, see Section 4.2. The solution is used to 
write the system (1) as

$$
\mathcal{H}\left\{\begin{aligned}
\dot{x} & =A x+B q+E w \\
z & =C x+D q+F w \\
u & =K x+L q+G w \\
q & =u-\operatorname{sat}(u)
\end{aligned}\right.
$$

where

$$
\left[\begin{array}{ccc}
A & B & E \\
C & D & F \\
K & L & G
\end{array}\right]=\left[\begin{array}{ccc}
\widetilde{A} & -\widetilde{B} & \widetilde{E} \\
\widetilde{C} & -\widetilde{D} & \widetilde{F} \\
0 & 0 & 0
\end{array}\right]+\left[\begin{array}{c}
\widetilde{B} \\
\widetilde{D} \\
I
\end{array}\right](I-\widetilde{L})^{-1}\left[\begin{array}{ccc}
\widetilde{K} & -\widetilde{L} & \widetilde{G}
\end{array}\right]
$$

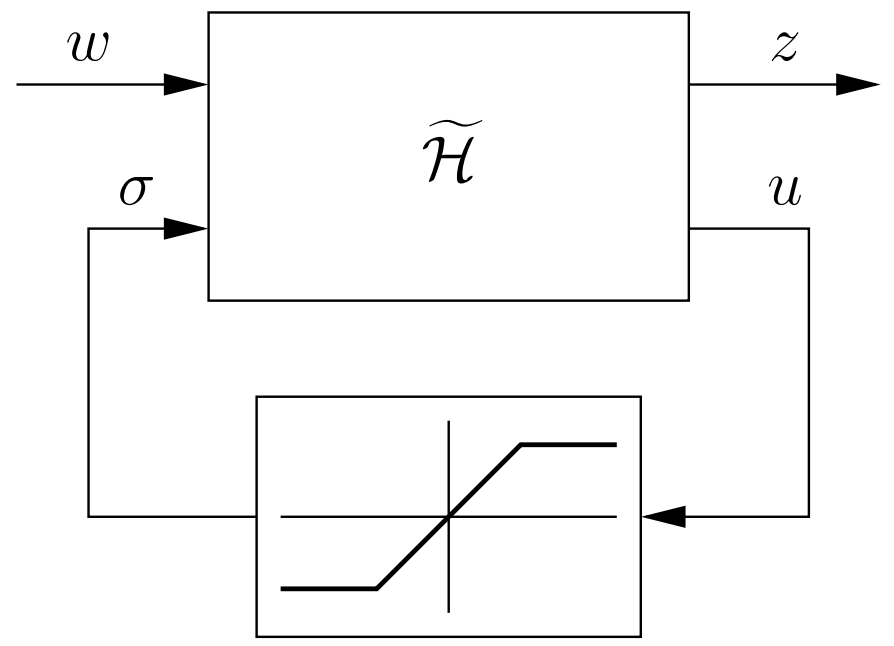

Figure 1: A closed-loop system involving a saturation nonlinearity.

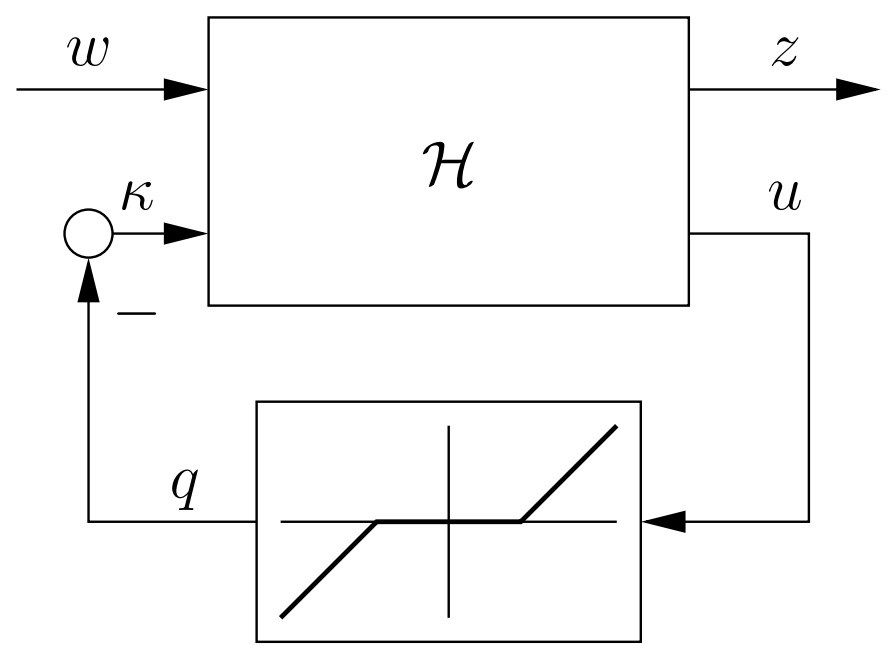

Figure 2: A closed-loop system involving a saturation nonlinearity written in compact form in negative feedback with a deadzone nonlinearity.

\subsection{Quadratic functions and semidefinite matrices}

The tools used here rely on nonnegative, quadratic functions for analysis. Such functions will lead to numerical algorithms that involve solving a set of linear matrix inequalities (LMIs) in order to certify internal stability or quantify external performance. Efficient commercial LMI solvers are widely available.

A nonnegative, quadratic function is a mapping $x \mapsto x^{T} P x$ where $P$ is symmetric, in other words, $P$ is equivalent to its transpose, and $x^{T} P x \geq 0$ for all $x$. In general, a symmetric matrix $P$ that satisfies $x^{T} P x \geq 0$ 
for all $x$ will be called a positive semidefinite matrix, written mathematically as $P \geq 0$. If $x^{T} P x>0$ for all $x \neq 0$, then $P$ will be called a positive definite matrix, written mathematically as $P>0$. A symmetric matrix $Q$ is negative semidefinite, written mathematically as $Q \leq 0$, if $-Q$ is positive semidefinite. A similar definition applies for a negative definite matrix. All of these terms are reserved for symmetric matrices. The reason for this is that a general square matrix $Z$ can be written as $Z=S+N$ where $S$ is symmetric and $N$ is anti-symmetric, i.e., $N=-N^{T}$ and then it follows that $x^{T} Z x=x^{T} S x$. In other words, the anti-symmetric part plays no role in determining the sign of $x^{T} Z x$. The notation $P_{1}>P_{2}$, respectively $P_{1} \geq P_{2}$, indicates that the matrix $P_{1}-P_{2}$ is positive definite, respectively positive semidefinite. Note that if $P>0$ then there exists $\varepsilon>0$ sufficiently small so that $P>\varepsilon I$.

\section{Unconstrained feedback systems}

To set the stage for results regarding the system (3), consider using quadratic functions to analyze unconstrained feedback systems, where the saturation nonlinearity in (3) is replaced by the identity function, in other words, $q \equiv 0$, so that the system (3) becomes

$$
\begin{aligned}
& \dot{x}=A x+E w \\
& z=C x+F w .
\end{aligned}
$$

\subsection{Internal stability}

When checking internal stability, $w$ is set to zero, $z$ plays no role, and (5) becomes simply $\dot{x}=A x$. To certify exponential stability for the origin of this system, one method that generalizes to feedback loops with input saturation involves finding a nonnegative, quadratic function that strictly decreases along solutions, except at the origin. Quadratic functions are convenient because they lead to stability tests that involve only linear algebra.

To determine whether a function is decreasing along solutions, it is enough to check whether, when evaluated along solutions, the function's time derivative is negative. For a continuously differentiable function, the time derivative can be obtained by computing the directional derivative of the function in the direction $A x$ and then evaluating this function along the solution. This corresponds to the mathematical equation

$$
\overbrace{V(x(t))}=\langle\nabla V(x(t)), A x(t)\rangle
$$

where $V$ represents the function, $\overbrace{V(x(t))}$ represents its time derivative along solutions at time $t, \nabla V(x)$ is the gradient of the function and $\langle\nabla V(x), A x\rangle$ is the directional derivative of the function in the direction $A x$. For a quadratic function $V(x)=x^{T} P x$, where $P$ is a symmetric matrix, the classical chain rule gives that this directional derivative equals

$$
x^{T}\left(P A+A^{T} P\right) x .
$$

Thus, in order for the time derivative to be negative along solutions, except at the origin, it should be the case that the directional derivative satisfies

$$
x^{T}\left(A^{T} P+P A\right) x<0 \quad \forall x \neq 0,
$$

in other words, $A^{T} P+P A<0$. In summary, to certify internal stability for the system (5) with a given matrix $A$, one looks for a symmetric matrix $P$ satisfying

$$
\begin{aligned}
P & \geq 0 \\
0 & >A^{T} P+P A .
\end{aligned}
$$

This is a particular example of a set of linear matrix inequalities (LMIs), which will be discussed in more detail in Section 3. Software for checking the feasibility of LMIs is widely available. It turns out that the LMIs in (6) are feasible if and only if the system (5) is internally stable. 


\subsection{External stability}

Now the external disturbance $w$ is no longer constrained to be zero. Thus, the relevant equation is (5). The goal is to determine the $\mathcal{L}_{2}$ gain from disturbance $w$ to the performance output variable $z$ and simultaneously establish internal stability. It is possible to give an arbitrarily tight upper bound on this gain by again exploiting nonnegative, quadratic functions. However, the quadratic function will not always be decreasing along solutions. Instead, an upper bound on the time derivative will be integrated to derive a relationship between the energy in the disturbance $w$ and the energy in the performance output variable $z$. Again, the directional derivative of the function $x^{T} P x$ in the direction $A x+E w$ generates the time derivative of the function along solutions. This time the directional derivative is given by

$$
x^{T}\left(A^{T} P+P A\right) x+2 x^{T} P E w .
$$

In order to guarantee an $\mathcal{L}_{2}$ gain less than a number $\gamma>0$ and to establish internal stability at the same time, it is sufficient to have

$$
x^{T}\left(A^{T} P+P A\right) x+2 x^{T} P E w<-\gamma\left(\frac{1}{\gamma^{2}} z^{T} z-w^{T} w\right) \quad \forall(x, w) \neq 0 .
$$

Using the definition of $z$ in (5), this condition is the same as having, for all $(x, w) \neq 0$,

$$
\left[\begin{array}{c}
x \\
w
\end{array}\right]^{T}\left(\left[\begin{array}{cc}
A^{T} P+P A & P E \\
E^{T} P & -\gamma I
\end{array}\right]+\frac{1}{\gamma}\left[\begin{array}{c}
C^{T} \\
F^{T}
\end{array}\right]\left[\begin{array}{ll}
C & F
\end{array}\right]\right)\left[\begin{array}{c}
x \\
w
\end{array}\right]<0 .
$$

In other words, the large matrix in the middle of this expression is negative definite. In summary, to certify internal stability and $\mathcal{L}_{2}$ external stability with gain less than $\gamma>0$ for the system (5) with a given set of matrices $(A, B, E, F)$, it suffices to find a symmetric matrix $P$ satisfying

$$
\begin{aligned}
P & \geq 0 \\
0 & >\left[\begin{array}{cc}
A^{T} P+P A & P E \\
E^{T} P & -\gamma I
\end{array}\right]+\frac{1}{\gamma}\left[\begin{array}{c}
C^{T} \\
F^{T}
\end{array}\right]\left[\begin{array}{ll}
C & F
\end{array}\right] .
\end{aligned}
$$

This is another set of LMIs, the feasibility of which can be tested with standard commercial software. Moreover, such software can be used to approximate the smallest possible number $\gamma$ that makes the LMIs feasible. The feasibility of the LMIs in (8) is also necessary for the $\mathcal{L}_{2}$ gain to be less than $\gamma$ with internal stability.

\section{$3 \quad$ Linear Matrix Inequalities}

As the preceding sections show, the analysis of dynamical systems benefits greatly from the availability of software to solve linear matrix inequalities (LMIs). The sections that follow show that LMIs also arise when using quadratic functions to analyze feedback loops with saturation. The goal of this section is to provide some familiarity with LMIs and to highlight some aspects to be aware of when using LMI solvers.

Linear matrix inequalities are generalizations of linear scalar inequalities. A simple example of a linear scalar inequality is

$$
2 z a+q<0
$$

where $a, q$ are known parameters and $z$ is a free variable. In contrast to the linear scalar equality $2 z a+q=0$, which either admits no solution (if $a=0$ and $q \neq 0$ ), an infinite number of solutions (if $a=0$ and $q=0$ ), or one solution (if $a \neq 0$ ), the linear scalar inequality (9) either admits no solutions (if $a=0$ and $q \geq 0$ ) or admits a convex set of solutions given by $\{z \in \mathbb{R}: z<-q /(2 a)\}$. In the former case the inequality is said to be infeasible. In the latter case it is said to be feasible.

Linear matrix inequalities generalize linear scalar inequalities by allowing the free variables to be matrices, allowing the expressions in which the free variables appear to be symmetric matrices, and generalizing negativity or positivity conditions to negative or positive definite matrix conditions. 
Replacing the quantities in (9) with their matrix counterparts and insisting that the resulting matrix is symmetric gives the linear matrix inequality

$$
A^{T} Z^{T}+Z A+Q<0 .
$$

In this LMI, $A$ and $Q$ are known matrices and $Q$ is symmetric. The matrix $Z$ comprises $m$ times $n$ free variables where $m$ denotes the number of columns of $Z$ and $n$ denotes the number of rows of $Z$. Characterizing the solution set of (10) is not as easy as before, because the solution space will be delimited by several hyperplanes that depend on the entries of the matrices $A$ and $Q$. However, one important property of this solution set is that it is convex. In particular, if the matrices $\left\{Z_{1}, \ldots, Z_{k}\right\}$ all satisfy the LMI (10) then for any set of numbers $\left\{\lambda_{1}, \ldots, \lambda_{k}\right\}$, where each of these numbers is between zero and one, inclusive, and the sum of the numbers is one, the matrix

$$
Z:=\sum_{i=1}^{k} \lambda_{i} Z_{i}
$$

also satisfies the LMI (10). The convexity property arises from the fact that (10) is linear in the free variable $Z$.

Now consider the case where $Q$ is taken to be zero and the free variable $Z$ is required to be symmetric and positive semidefinite. The variable $Z$ will be replaced by the variable $P$ for this case. With the free variable being symmetric, the parameter $A$ is now required to be a square matrix. Now, recall that the matrix condition $A^{T} P+P A<0$ is equivalent to the existence of $\varepsilon>0$ such that $A^{T} P+P A+\varepsilon I \leq 0$. Therefore, an extra free variable $\varepsilon$ can be introduced to write the overall conditions as the single LMI

$$
\left[\begin{array}{cc}
P & 0 \\
0 & -\left(A^{T} P+P A+\varepsilon I\right)
\end{array}\right] \geq 0 .
$$

The implicit constraint that $P$ is symmetric reduces the number of free variables in the matrix $P$ to the quantity $n(n+1) / 2$ where $n$ is the size of the square matrix $P$. The feasibility of the LMI (11) is equivalent to the simultaneous feasibility of the two LMIs

$$
\begin{aligned}
& P \geq 0 \\
& 0>A^{T} P+P A
\end{aligned}
$$

which match the LMIs (6) that appeared in the analysis of internal stability for linear systems. It is worth noting that most LMI solvers have difficulty with inequalities that are not strict. This is because sometimes, in this case, the feasibility is not robust to small changes in the parameters of the LMI. For example, with the choice

$$
A=\left[\begin{array}{cc}
0 & 1 \\
-1 & 0
\end{array}\right]
$$

the LMIs $P>0$ and $0 \geq A^{T} P+P A$ are feasible (note that the strictly inequality and the nonstrict inequality have been exchanged relative to (12)) as can be seen by taking $P=I$. However, the LMIs are not feasible if one adds to $A$ the matrix $\varepsilon I$ for any $\varepsilon>0$. On the other hand, strict LMIs, if feasible, are always robustly feasible. Fortunately, the feasibility of the LMIs (12) is equivalent to the feasibility of the LMIs

$$
\begin{aligned}
P & >0 \\
0 & >A^{T} P+P A .
\end{aligned}
$$

This can be verified by letting $\widehat{P}$ denote a feasible solution to (12) and observing that $\widehat{P}+\varepsilon I$ must be a feasible solution to (13) for $\varepsilon>0$ sufficiently small. From the discussion in Section 2.1, it follows that the LMIs (13) are feasible if and only if the system $\dot{x}=A x$ is internally stable.

Next consider the matrix conditions that appeared in Section 2.2, the feasibility of which was equivalent to having $\mathcal{L}_{2}$ external stability with gain less than $\gamma>0$ and internal stability for (5). Using the same idea 
as above to pass to a strict inequality for the matrix $P$, the feasibility of the matrix conditions in $(8)$ is equivalent to the feasibility of the matrix conditions

$$
\begin{aligned}
P & >0 \\
0 & >\left[\begin{array}{cc}
A^{T} P+P A & P E \\
E^{T} P & -\gamma I
\end{array}\right]+\frac{1}{\gamma}\left[\begin{array}{c}
C^{T} \\
F^{T}
\end{array}\right]\left[\begin{array}{ll}
C & F
\end{array}\right] .
\end{aligned}
$$

The matrices $(A, E, C, F)$ are parameters that define the problem. If the value $\gamma$ is specified, then the feasibility of the resulting LMIs in terms of the free variable $P$ can be checked with an LMI solver. If the interest is in finding values for $\gamma>0$ to make the matrix conditions feasible, then $\gamma$ can be taken to be a free variable. However, the matrix conditions do not constitute LMIs because of the nonlinear dependence on the free parameter $\gamma$ through the factor $1 / \gamma$ that appears. Fortunately, there is a way to convert the matrix conditions above into LMIs in the free variables $\gamma$ and $P$ using the following fact:

(Schur complements) Let $Q$ and $R$ be symmetric matrices and let $S$ have the same number of rows as $Q$ and the same number of columns as $R$. Then the matrix condition

$$
\left[\begin{array}{cc}
Q & S \\
S^{T} & R
\end{array}\right]>0
$$

is equivalent to the matrix conditions

$$
\begin{aligned}
R & >0 \\
Q-S R^{-1} S^{T} & >0
\end{aligned}
$$

This fact can be applied to the matrix conditions (14) to obtain the matrix conditions

$$
\begin{aligned}
\gamma & >0 \\
P & >0 \\
0 & >\left[\begin{array}{ccc}
A^{T} P+P A & P E & C^{T} \\
E^{T} P & -\gamma I & F^{T} \\
C & F & -\gamma I
\end{array}\right]
\end{aligned}
$$

which are LMIs in the free variables $P$ and $\gamma$. If the system $\dot{x}=A x$ is internally stable then these LMIs will be feasible. This follows from the fact that there will exist $P>0$ satisfying $A^{T} P+P A<0$, which is the matrix that appears in the upper left-hand corner of the large matrix in (15), and a consequence of Finsler's Lemma, which is the following:

(Finsler's Lemma) Let $Q$ be symmetric and let $H$ have the same number of columns as $Q$. If $\zeta^{T} Q \zeta<0$ for all $\zeta \neq 0$ satisfying $H \zeta=0$ then, for all $\gamma>0$ sufficiently large, $Q-\gamma H^{T} H<0$.

If one applies this fact with the matrix

$$
Q:=\left[\begin{array}{ccc}
A^{T} P+P A & P E & C^{T} \\
E^{T} P & 0 & F^{T} \\
C & F & 0
\end{array}\right]
$$

and

$$
H=\left[\begin{array}{lll}
0 & 0 & 0 \\
0 & I & 0 \\
0 & 0 & I
\end{array}\right]
$$

one sees that the LMIs in (15) will be feasible for an appropriate $P$ matrix and large enough $\gamma>0$.

To determine a tight upper bound on the $\mathcal{L}_{2}$ gain, one is interested in making $\gamma$ as small as possible. The task of minimizing $\gamma$ subject to satisfying the LMIs (15) is an example of an LMI eigenvalue problem 
and can be written as:

$$
\begin{aligned}
\min _{P, \gamma} \gamma, & \text { subject to } \\
P>0 & \\
0> & {\left[\begin{array}{ccc}
A^{T} P+P A & P E & C^{T} \\
E^{T} P & -\gamma I & F^{T} \\
C & F & -\gamma I
\end{array}\right] . }
\end{aligned}
$$

Since large block matrices that appear in LMIs must always be symmetric, the entries below the diagonal must be equal to the transposes of the entries above the diagonal. For this reason, such matrices can be replaced with the ' $\star$ ' symbol without any loss of information. For example, (16) can be written as

$$
\begin{aligned}
\min _{P, \gamma} \gamma, & \text { subject to } \\
P & >0, \\
0 & >\left[\begin{array}{ccc}
A^{T} P+P A & P E & C^{T} \\
\star & -\gamma I & F^{T} \\
\star & \star & -\gamma I
\end{array}\right] .
\end{aligned}
$$

with no loss of information.

An alternative notation that may simplify (16) relies on the use of the function "He" which, given any square matrix $X$ is defined as $\operatorname{He} X:=X+X^{T}$, so that (16) can be written as

$$
\begin{aligned}
\min _{P, \gamma} \gamma, \quad \text { subject to } \\
\quad P>0 \\
0>\operatorname{He}\left[\begin{array}{ccc}
P A & P E & 0 \\
0 & -\gamma I / 2 & 0 \\
C & F & -\gamma I / 2
\end{array}\right] .
\end{aligned}
$$

The LMI feasibility and eigenvalue problems can be solved efficiently using modern numerical software packages. As an example, the code needed in MATLAB's LMI control toolbox to implement the search for the optimal solution to (16) or, equivalently, of (17) and (18) is given next.

Example 1 Implementing LMIs using the MATLAB's LMI control toolbox requires first defining the LMI constraints structure and then running the solver on those constraints. The LMI constraints are specified by a start line (setlmis([]);) and an end line (mylmisys=getlmis;) which also gives a name to the LMI constraints. Then the LMI constraints consist of a first block where the LMI variables are listed and of a second block where the LMI constraints are described in terms of those variables. The following code gives a rough idea of how this should be implemented. Comments within the code provides indications of where the different blocks are located. For more details, the reader should refer to the MATLAB's LMI control toolbox user's guide.

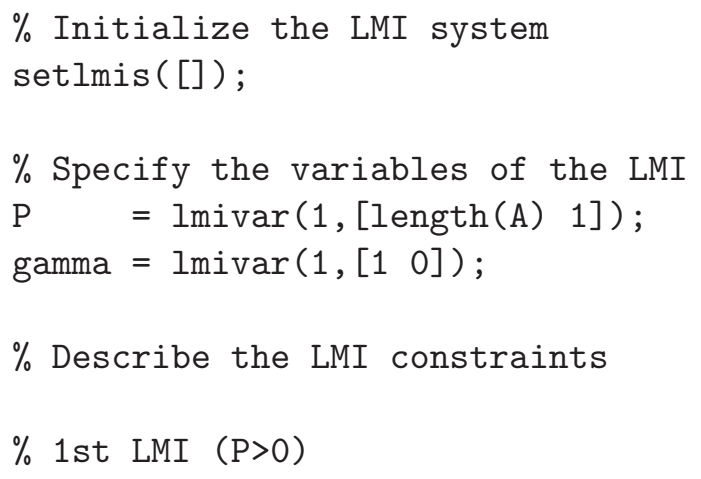




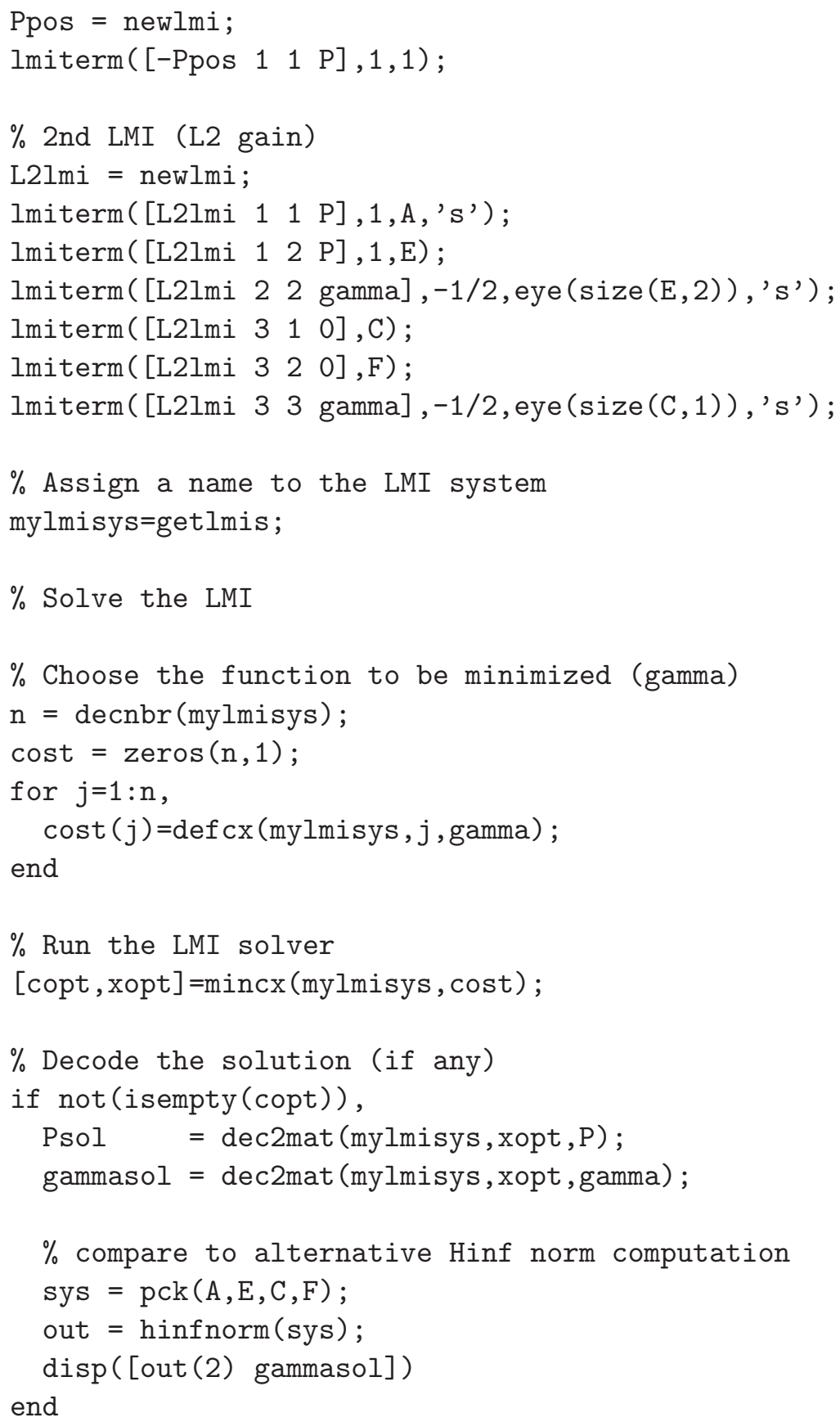

Example 2 The MATLAB code illustrated in the previous example is quite streamlined and using the LMI control toolbox in such a direct way can many times become quite complicated in terms of actual MATLAB code. An alternative to this is to indirectly specify the LMI constraints and use the LMI control toolbox solver by way of the YALMIP (=Yet Another LMI Parser) front-end. The advantages of using YALMIP mainly reside in the increased simplicity of the code (thereby significantly reducing the probability of typos) and in the code portability to alternative solvers to the classical LMI control toolbox (SeDuMi is a much used alternative). The same calculation reported in the previous example is computed using the YALMIP front-end in the following code:

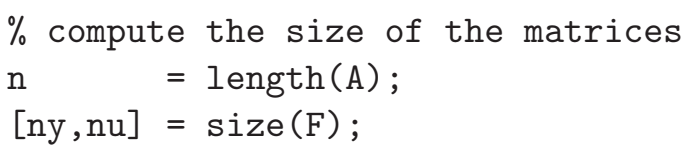




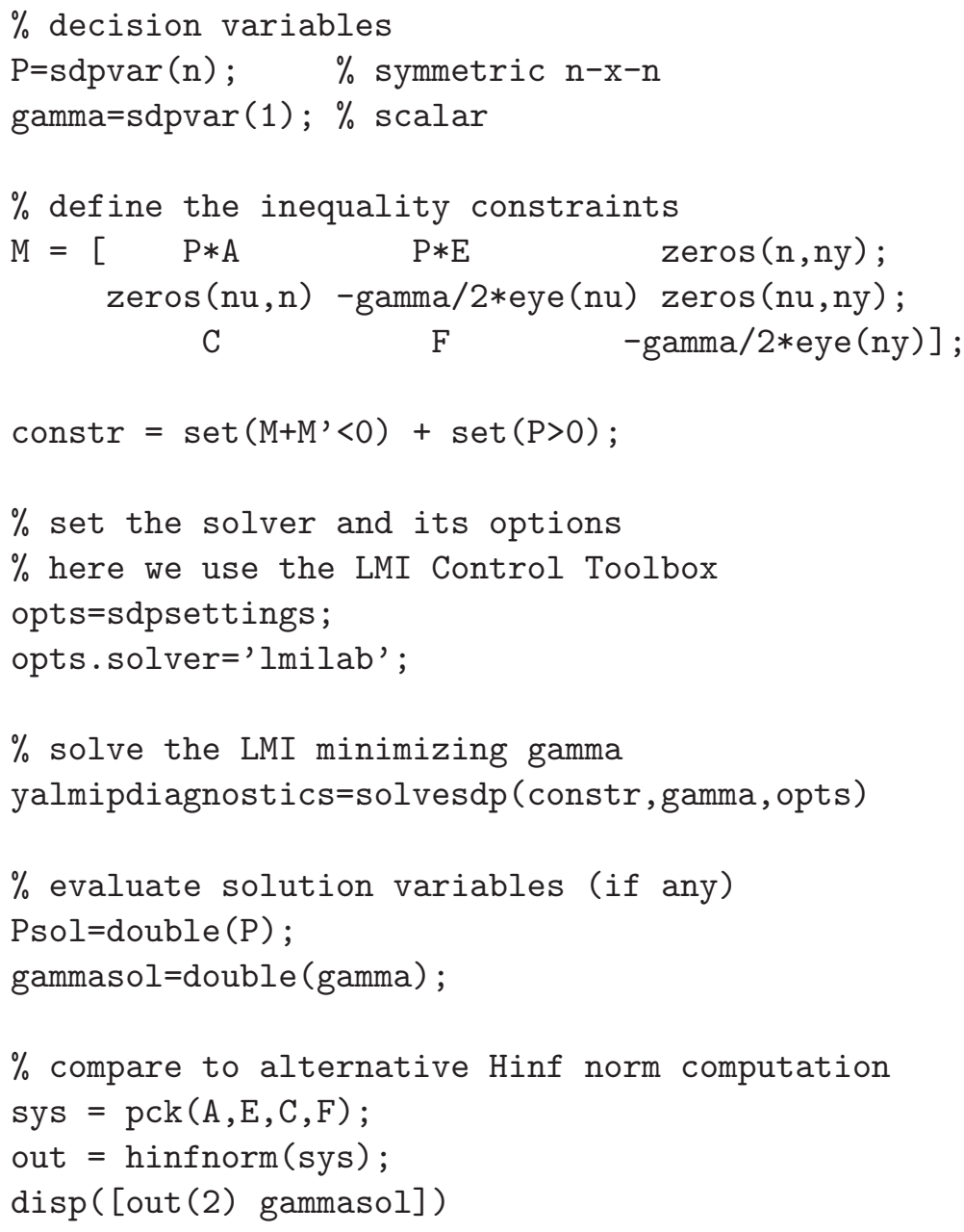

Example 3 CVX is another useful program for solving structured convex optimization problems, including LMIs. The previous calculations in CVX are as follows:

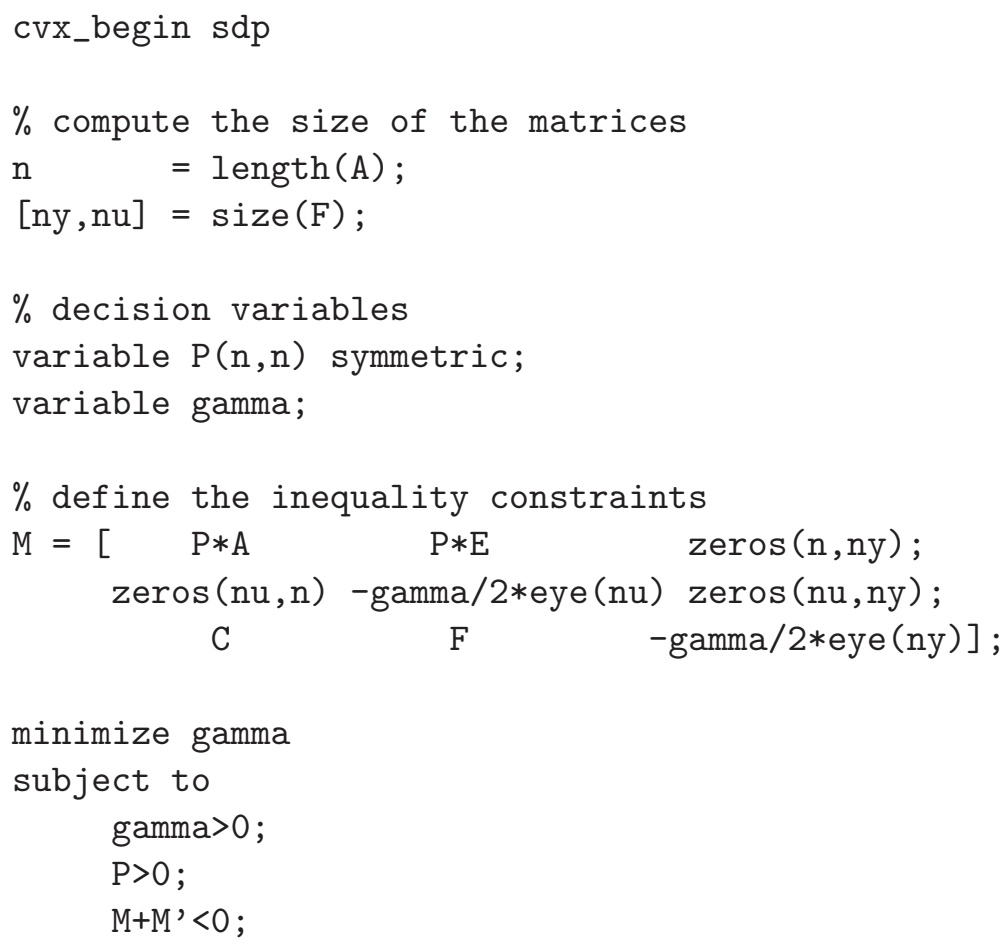


There are a few points to make about the variability in solutions to LMI eigenvalue problems returned by using different commercial solvers. First, notice that the LMI eigenvalue problem in (17) involves an optimization over an open set of matrices. So, technically, it is not possible to achieve the minimum. It would be more appropriate to say that the optimization problem is looking for the infimum. Indeed, if a minimum $\gamma^{*}$ existed and satisfied the LMIs then it would also be the case that $\gamma-\varepsilon$ satisfied the LMIs for $\varepsilon>0$ sufficiently small, contradicting the fact that $\gamma^{*}$ is a minimum. A consequence of this fact is that, since it is not possible to reach an infimum, each solver will need to make its own decision about the path to take toward the infimum and at what point to stop. Different paths to the infimum and different stopping conditions will cause different solvers to return different solutions. Second, unless the optimization is strictly convex, the solution to the optimization problem may not be unique. This fact may also contribute to variability in the solutions returned by different solvers. In each of these cases, the different minima returned should be quite close to one another, whereas the matrices returned that satisfy the LMIs may be quite different.

Before moving on to the analysis of constrained feedback systems, one additional matrix manipulation, which will be used to analyze systems with saturation, will be introduced. It is closely related to Finsler's Lemma.

(S-procedure) Let $M_{0}$ and $M_{1}$ be symmetric matrices and suppose there exists $\zeta^{*}$ such that $\zeta^{*} M_{1} \zeta^{*}>0$. Then the following statements are equivalent:

i. There exists $\tau>0$ such that $M_{0}-\tau M_{1}>0$.

ii. $\zeta^{T} M_{0} \zeta>0$ for all $\zeta \neq 0$ such that $\zeta^{T} M_{1} \zeta \geq 0$.

The implication from (i) to (ii) is simple to see, and does not require the existence of $\zeta^{*}$ such that $\zeta^{*} M_{1} \zeta^{*}>0$. The opposite implication is nontrivial.

\section{Constrained feedback systems: global analysis}

\subsection{Sector characterizations of nonlinearities}

In order to arrive at LMIs when checking the internal stability and $\mathcal{L}_{2}$ external stability for feedback loops with saturations or deadzones, one typically inscribes the saturation or deadzone into a conic region and applies the S-procedure. To understand the idea behind inscribing a nonlinearity into a conic region, consider a scalar saturation function. Figure 3 contains, on the left, the block diagram of the saturation function and, on the right, the graph of the saturation function. The figure emphasizes that the graph of the saturation function is contained in a conic sector delimited by the line passing through the origin with slope zero and the line passing through the origin with slope one. The deadzone nonlinearity, as shown in Figure 4, is also contained in this sector. In fact, the sector contains any scalar nonlinearity with the property that its output $y$ always has the same sign as its input $u$ and $y$ has a magnitude that is never bigger than that of $u$. This condition can be expressed mathematically using the quadratic inequality $y(u-y) \geq 0$, which, when focusing on the deadzone nonlinearity where $y=q$, becomes

$$
q(u-q) \geq 0 .
$$

This condition says that $q u \geq q^{2}$, which captures the sign and magnitude information described above.

For a decentralized nonlinearity where each component of the nonlinearity is inscribed in the sector described above, the quadratic condition

$$
\sum_{i=1}^{n_{u}} w_{i} q_{i}\left(u_{i}-q_{i}\right) \geq 0
$$




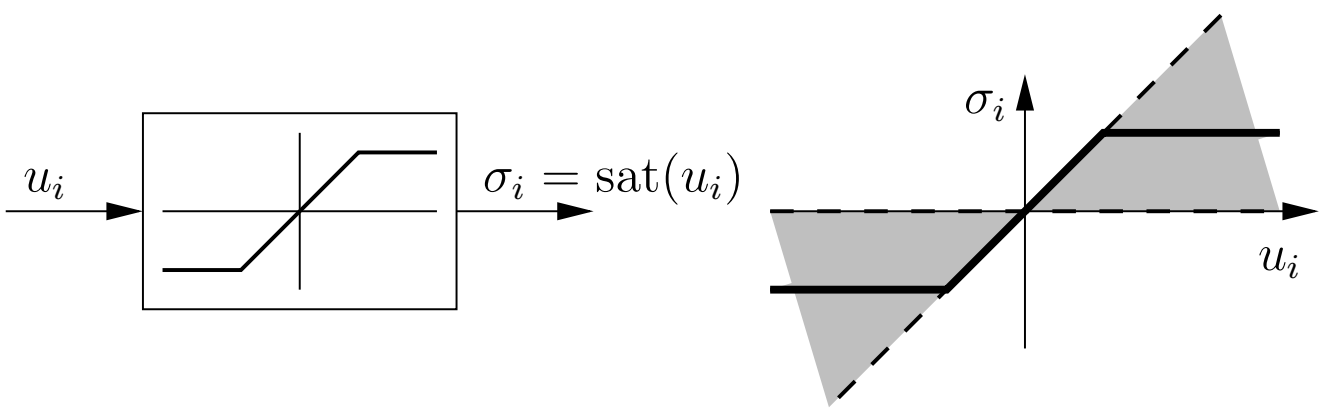

Figure 3: The scalar saturation function and its sector properties.
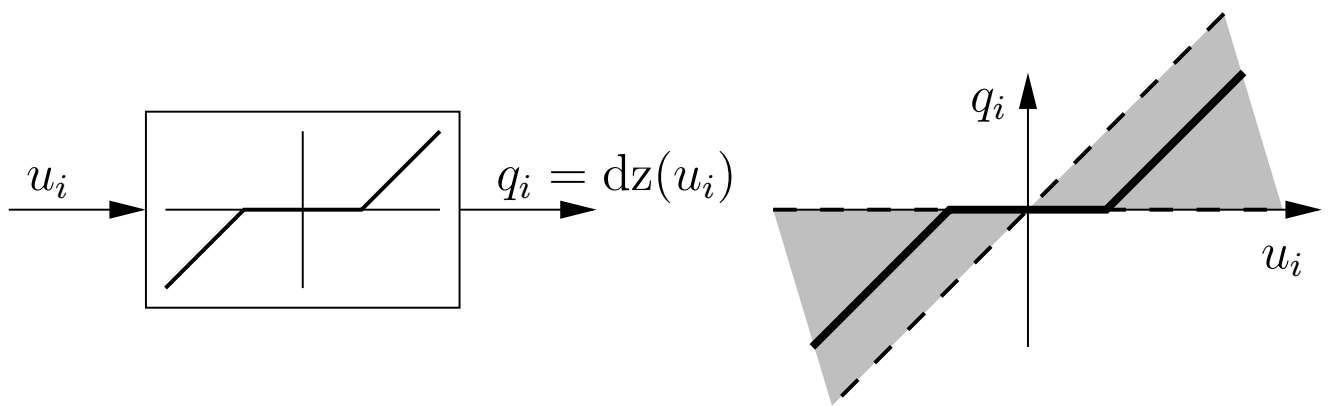

Figure 4: The scalar deadzone function and its sector properties.

holds, where $q_{i}$ are the components of the output vector $y, u_{i}$ are the components of the input vector $u$ and $w_{i}$ are arbitrary positive weightings. This can also be written as

$$
q^{T} W(u-q) \geq 0
$$

where $W$ is a diagonal matrix consisting of the values $w_{i}$.

A sector characterization of nonlinearities introduces some conservativeness since the analysis using sectors will apply to any nonlinearity inscribed in the sector. The payoff in using sector characterizations is that the mathematical description, in terms of quadratic inequalities, is compatible with the analysis of feedback systems using quadratic functions. Indeed, the S-procedure described earlier permits combining the quadratic inequality describing the sector with the quadratic inequalities involved in the directional derivative to arrive at LMIs for the analysis of feedback loops with sector nonlinearities. This will be done subsequently.

\subsection{Guaranteeing well-posedness}

Before analyzing the feedback loop given by (3), shown in Figure 2, it must be established that the feedback loop is well posed. In particular, it should be verified that the equation

$$
u-L(u-\operatorname{sat}(u))=v
$$

admits a solution $u$ for each $v$ and that the solution $u(v)$ is a sufficiently regular function of $v$. When sat $(u)$ is decentralized, the equation admits a solution that is a Lipschitz function of $v$ when each of the matrices

$$
I-L \Delta \quad \Delta=\operatorname{diag}\left(\delta_{1}, \ldots, \delta_{n_{u}}\right), \quad \delta_{i} \in\{0,1\} \quad \forall i \in\left\{1, \ldots, n_{u}\right\}
$$

is invertible. It turns out that each of these matrices is invertible when there exists a diagonal, positive definite matrix $W$ such that

$$
L^{T} W+W L-2 W<0 .
$$

This LMI in $W$ will appear naturally in the analysis LMIs in the following sections. The LMI can be strengthened by replacing the ' 2 ' with a smaller positive number in order to guarantee that the feedback 
loop is not too close to being ill posed. In particular, in many of the algorithms proposed later in this book, the following LMI will be employed,

$$
L^{T} W+W L-2(1-\nu) W<0,
$$

or equivalent versions of it. This LMI is be referred to as strong well-posedness constraint and enforces a bound on the speed of variation of the closed-loop state (by bounding the Lipschitz constant of the right hand side of the dynamic equation).

\subsection{Internal stability}

To analyze internal stability for (3), set $w=0$ and consider the resulting system, which is given by

$$
\begin{aligned}
\dot{x} & =A x+B q \\
z & =C x+D q \\
u & =K x+L q \\
q & =u-\operatorname{sat}(u) .
\end{aligned}
$$

Again, a nonnegative, quadratic function $V(x)=x^{T} P x$ will be used. Like in the case of linear systems, it is desirable for the time derivative of $V(x(t))$ to be negative except at the origin. The time derivative is obtained from the directional derivative of $V(x)$ in the direction $A x+B q$, which is given by

$$
\langle\nabla V(x), A x+B q\rangle=x^{T}\left(A^{T} P+P A\right) x+2 x^{T} P B q .
$$

Since $q$ is the output of the deadzone nonlinearity, it follows from the discussion in Section 4.1 that the property

$$
q^{T} W(u-q) \geq 0
$$

holds for any diagonal positive semidefinite matrix $W$. Then, using the definition of $u$ in (22), the requirement on the time derivative translates to the condition

$$
q^{T} W(K x+L q-q) \geq 0,(x, q) \neq 0 \quad \Longrightarrow \quad x^{T}\left(A^{T} P+P A\right) x+2 x^{T} P B q<0 .
$$

In order to guarantee this implication, it is enough to check that

$$
x^{T}\left(A^{T} P+P A\right) x+2 x^{T} P B q+2 q^{T} W(K x+L q-q)<0 \quad \forall(x, q) \neq 0 .
$$

This is the easy implication in the S-procedure, where the $\tau$ has been absorbed into the free variable $W$. In fact, the S-procedure can be used to understand that there is no loss of generality in replacing (24) by (25). The condition (25) can be written equivalently as

$$
\left[\begin{array}{l}
x \\
q
\end{array}\right]^{T}\left[\begin{array}{cc}
A^{T} P+P A & P B+K^{T} W \\
B^{T} P+W K & W L+L^{T} W-2 W
\end{array}\right]\left[\begin{array}{c}
x \\
q
\end{array}\right]<0 \quad \forall(x, q) \neq 0 .
$$

In other words,

$$
\begin{aligned}
0 & >\left[\begin{array}{cc}
A^{T} P+P A & P B+K^{T} W \\
\star & W L+L^{T} W-2 W
\end{array}\right] \\
& =\operatorname{He}\left[\begin{array}{cc}
P A & P B \\
W K & W L-W
\end{array}\right] .
\end{aligned}
$$

This condition is an LMI in the free variables $P$, which should be positive semidefinite, and $W$, which should be diagonal and positive definite. The matrices $(A, B, K, L)$ are parameters that define the system (22). Notice that, because of the lower right-hand entry in the matrix, the LMI condition for well-posedness given in Section 4.2 is automatically guaranteed when (27) is satisfied. In addition, because of the upper left-hand 
entry in the matrix, the matrix $A$ must be such that the linear system $\dot{x}=A x$ is internally stable. Finally, note that the LMI condition is not necessary for internal stability. For example, consider the system

$$
\begin{aligned}
\dot{x} & =-x+q \\
u & =x \\
q & =u-\operatorname{sat}(u) .
\end{aligned}
$$

The LMI for internal stability becomes

$$
0>\left[\begin{array}{cc}
-2 p & -p-w \\
\star & -2 w
\end{array}\right]
$$

or, equivalently,

$$
H e\left[\begin{array}{ll}
p & w \\
p & w
\end{array}\right]>0
$$

In order for this matrix to be positive definite, the determinant of the matrix, given by $4 p w-(p+w)^{2}$, must be positive. However, $4 p w-(p+w)^{2}=-(p-w)^{2}$ which is never positive. Therefore, the internal stability LMI is not feasible. Nevertheless, the system is internally stable since the system is equivalent to the system

$$
\dot{x}=-\operatorname{sat}(x)
$$

for which the quadratic function $V(x)=x^{2}$ decreases along solutions but not at a quadratic rate.

In general, the LMI for internal stability for the system (3) will not be feasible if the linear system $\dot{x}=\left[A+B(I-L)^{-1} K\right] x$ is not internally stable. This is the system that results from (3) by setting $w=0$ and $\operatorname{sat}(u) \equiv 0$. To put it another way, when the system (3) is expressed as a feedback interconnection of a linear system with a saturation nonlinearity rather than a deadzone, the resulting system is

$$
\begin{aligned}
\dot{x} & =\left[A+B(I-L)^{-1} K\right] x-B\left[I+(I-L)^{-1} L\right] \sigma+\left[E+B(I-L)^{-1} G\right] w \\
z & =\left[C+D(I-L)^{-1} K\right] x-D\left[I+(I-L)^{-1} L\right] \sigma+\left[F+D(I-L)^{-1} G\right] w \\
u & =(I-L)^{-1}[K x-L \sigma+G w] \\
\sigma & =\operatorname{sat}(u) .
\end{aligned}
$$

Then, for the LMI-based internal stability test in (27) to be feasible, it is necessary that (32) with $\sigma=0$ and $w=0$ is internally stable.

\subsection{External stability}

Now consider establishing $\mathcal{L}_{2}$ external stability for the system (3). Again relying on a nonnegative, quadratic function, and combining the ideas in Sections 2.2 and 4.3, it is sufficient to have that

$$
q^{T} W(G w+K x+L q-q) \geq 0,(x, q, w) \neq 0
$$

implies

$$
x^{T}\left(A^{T} P+P A\right) x+2 x^{T} P(B q+E w)<-\gamma\left(\frac{1}{\gamma^{2}} z^{T} z-w^{T} w\right) .
$$

Then, using the definition of $z$, and applying the S-procedure and Schur complements, produces the condition

$$
\left[\begin{array}{cccc}
A^{T} P+P A & P B+K^{T} W & P E & C^{T} \\
\star & -2 W+W L+L^{T} W & W G & D^{T} \\
\star & \star & -\gamma I & F^{T} \\
\star & \star & \star & -\gamma I
\end{array}\right]<0
$$

which is an LMI in the unknowns $P=P^{T}>0, W>0$ diagonal and $\gamma>0$. The four blocks in the upper left corner correspond to the LMI for internal stability given in (27). It follows that if the system (3) is internally stable then the LMI (34) is feasible using the solutions $P$ and $W$ from the internal stability LMI 
and then picking $\gamma>0$ sufficiently large. Of course, the goal is to see how small $\gamma$ can be chosen. This objective corresponds to solving the eigenvalue problem

$$
\begin{aligned}
\min _{P, W, \gamma} \gamma, \quad \text { subject to } \\
P=P^{T}>0, W>0 \text { diagonal } \\
\operatorname{He}\left[\begin{array}{cccc}
P A & P B & P E & 0 \\
W K & W L-W & W G & 0 \\
0 & 0 & -\gamma I / 2 & 0 \\
C & D & F & -\gamma I / 2
\end{array}\right]<0 .
\end{aligned}
$$

Since a necessary condition for global internal and external stability for the system (3) is that the matrix $A+B(I-L)^{-1} K$ is exponentially stable, there will be many situations where the LMIs given in this section will not be feasible. For this reason, it is helpful to have a generalization of these results for the case where only regional internal and external stability can be established. This is the topic of the next section.

\section{Constrained feedback systems: regional analysis}

\section{$5.1 \quad$ Regional sectors}

To produce LMI results that are helpful for a regional analysis of systems with saturation, it is necessary to come up with a tighter sector characterization of the saturation nonlinearity while still using quadratic inequalities. This appears to be impossible to do globally. However, for analysis over a bounded region, there is a way to make progress. For the scalar saturation function, one fruitful idea is to take $H$ to be an arbitrary row vector and note that the quadratic inequality

$$
(\sigma+H x)(u-\sigma) \geq 0 \quad \forall x \text { satisfying } \quad \operatorname{sat}(H x)=H x
$$

will hold for any input/output pairs $u$ and $\sigma$ generated by the saturation nonlinearity. This can be verified by checking two cases:

i. If $\sigma:=\operatorname{sat}(u)=u$ then $\sigma=u$ and so the quadratic form on the left-hand side is zero.

ii. If $\operatorname{sat}(u) \neq u$ then the sign of $(u-\sigma)$ is equal to the sign of $u$ and also the sign of $\sigma+H x$ is equal to the sign of $u$ so that the product is not negative. The condition $\operatorname{sat}(H x)=H x$ is used in this step.

The corresponding condition for the deadzone nonlinearity having input $u$ and output $q$ can be derived from (36) by using the definition $q:=\mathrm{dz}(u)=u-\operatorname{sat}(u)=u-\sigma$. The resulting quadratic condition is

$$
(u-q+H x) q \geq 0 \quad \forall x \text { satisfying } \quad \operatorname{sat}(H x)=H x .
$$

The decentralized vector version of this inequality is

$$
(u-q+H x)^{T} W q \geq 0 \quad \forall x \text { satisfying } \quad \operatorname{sat}(H x)=H x
$$

where $H$ is now a matrix of appropriate dimensions and $W$ is a diagonal, positive definite matrix. In order to have $\operatorname{sat}(H x)=H x$ for all possible values of $x$, it must be the case that $H=0$. In this case, the sector condition in (38) reduces to the global sector condition used previously.

\subsection{Internal stability}

In this section, the sector characterization of the previous section is exploited. The saturation nonlinearity is taken to be decentralized, the saturation limits are taken to be symmetric, and the $i$ th function is limited 
in range to $\pm \bar{u}_{i}$. In order to guarantee the condition $\operatorname{sat}(H x(t))=H x(t)$ for solutions to be considered, which is needed to exploit the sector condition of the previous section, the condition

$$
x^{T} H_{i}^{T} H_{i} x / \bar{u}_{i}^{2}<x^{T} P x \quad \forall x \neq 0
$$

is imposed, where $H_{i}$ denotes the $i$-th row of $H$. Using Schur complements, the condition (39) can be written as the matrix condition

$$
\left[\begin{array}{cc}
P & H_{i}^{T} \\
H_{i} & \bar{u}_{i}^{2}
\end{array}\right]>0, \quad i=1, \ldots, n_{u} .
$$

According to (39), $x^{T} P x \leq 1$ implies $\operatorname{sat}(H x)=H x$. So, the analysis of internal stability can now proceed like before but restricting attention to values of $x$ for which $x^{T} P x \leq 1$ and using the sector condition from the previous section. Indeed, if in the set $\mathcal{E}(P):=\left\{x: x^{T} P x \leq 1\right\}$ the quadratic function $x^{T} P x$ is decreasing along solutions, then the set $\mathcal{E}$ will be forward invariant and convergence to the origin will ensue.

Picking up the analysis from Section 4.3 immediately after the description of the directional derivative of the quadratic function $x^{T} P x$ in the direction $A x+B q$ in equation (23), the condition (24) is now replaced by the condition

$$
\begin{aligned}
& q^{T} W(H x+K x+L q-q) \geq 0,(x, q) \neq 0, \\
& \Longrightarrow \quad x^{T}\left(A^{T} P+P A\right) x+2 x^{T} P B q<0 .
\end{aligned}
$$

In order to guarantee this implication, it is enough to check that, for all $(x, q) \neq 0$,

$$
x^{T}\left(A^{T} P+P A\right) x+2 x^{T} P B q+2 q^{T} W(H x+K x+L q-q)<0 .
$$

The condition (42) can be written equivalently as

$$
\left[\begin{array}{l}
x \\
q
\end{array}\right]^{T}\left[\begin{array}{cc}
A^{T} P+P A & P B+K^{T} W+H^{T} W \\
B^{T} P+W K+W H & W L+L^{T} W-2 W
\end{array}\right]\left[\begin{array}{c}
x \\
q
\end{array}\right]<0 .
$$

In other words,

$$
0>\operatorname{He}\left[\begin{array}{cc}
P A & P B \\
W H+W K & W L-W
\end{array}\right] .
$$

The matrix conditions that can then be used to establish internal stability over the region $\mathcal{E}(P)$ are given by (40) and (44) together with $P>0, W>0$ and the condition that $W$ is diagonal. These conditions are not linear in the free variables $P, W$ and $H$. In particular, notice the term $H^{T} W$ that appears in the upper right-hand corner of the matrix in (44). Nevertheless, there is a nonlinear transformation of the free variables that results in an LMI condition. The transformation exploits the fact that the condition $S<0$ is equivalent to the condition $R^{T} S R<0$ for any invertible matrix $R$. Make the definitions $Q:=P^{-1}$, $U:=W^{-1}$, and $Y:=H Q$. These definitions make sense since $W$ and $P$ must be positive definite. Moreover, $P, W$ and $H$ can be recovered from $Q, U$ and $Y$. Now observe that

$$
\left[\begin{array}{cc}
P^{-1} & 0 \\
0 & I
\end{array}\right]\left[\begin{array}{cc}
P & H_{i}^{T} \\
H_{i} & \bar{u}_{i}^{2}
\end{array}\right]\left[\begin{array}{cc}
P^{-1} & 0 \\
0 & I
\end{array}\right]=\left[\begin{array}{cc}
Q & Q H_{i}^{T} \\
H_{i} Q & \bar{u}_{i}^{2}
\end{array}\right]=\left[\begin{array}{cc}
Q & Y_{i}^{T} \\
Y_{i} & \bar{u}_{i}^{2}
\end{array}\right]
$$

and

$$
\begin{aligned}
{\left[\begin{array}{cc}
P^{-1} & 0 \\
0 & W^{-1}
\end{array}\right]\left[\begin{array}{cc}
A^{T} P+P A & P B+K^{T} W+H^{T} W \\
\star & W L+L^{T} W-2 W
\end{array}\right]\left[\begin{array}{cc}
P^{-1} & 0 \\
0 & W^{-1}
\end{array}\right] } \\
=\left[\begin{array}{cc}
Q A^{T}+A Q & B U+Q K^{T}+Q H^{T} \\
\star & L U+U L^{T}-2 U
\end{array}\right] \\
=\left[\begin{array}{cc}
Q A^{T}+A Q & B U+Q K^{T}+Y^{T} \\
\star & L U+U L^{T}-2 U
\end{array}\right] .
\end{aligned}
$$


Thus, internal stability over the region $\mathcal{E}\left(Q^{-1}\right)$ results if the following LMIs are feasible in the free parameters $Q, U$ and $Y$ :

$$
\begin{aligned}
Q & >0 \\
U & >0 \text { diagonal } \\
0 & >\operatorname{He}\left[\begin{array}{cc}
A Q & B U \\
Y+K Q & L U-U
\end{array}\right] \\
{\left[\begin{array}{cc}
Q & Y_{i}^{T} \\
Y_{i} & \bar{u}_{i}^{2}
\end{array}\right] } & >0, \quad i=1, \ldots, n_{u} .
\end{aligned}
$$

Moreover, it is possible to use these LMIs as constraints for optimizing the set $\mathcal{E}\left(Q^{-1}\right)$ in some way. In the next section, LMIs will be given for establishing internal stability and minimizing the $\mathcal{L}_{2}$ external gain over a region. Numerical examples will be given there.

\subsection{External stability}

Consider establishing $\mathcal{L}_{2}$ external stability for the system (3) over a region. The initial condition will be taken to be zero and the size of the solution $x(t)$ will be limited by limiting the energy in the disturbance input $w$. In particular, if it is true that

$$
\dot{V}(x(t)) \leq w^{T}(t) w(t)
$$

whenever $x(t)^{T} P x(t) \leq s^{2}$ and if $\|w\|_{2} \leq s$ then it follows by integrating this inequality that

$$
x(t)^{T} P x(t)=V(x(t)) \leq\|w\|_{2}^{2} \leq s^{2} \quad \forall t \geq 0 .
$$

Thus, changing the bound in (39) to

$$
s^{2} x^{T} H_{i}^{T} H_{i} x / \bar{u}_{i}^{2}<x^{T} P x \quad \forall x \neq 0
$$

will guarantee that $\operatorname{sat}(H x(t))=H x(t)$ for all disturbances $w$ with $\|w\|_{2} \leq s$. The condition (50) corresponds to the matrix condition

$$
\left[\begin{array}{cc}
P & H_{i}^{T} \\
H_{i} & \bar{u}_{i}^{2} / s^{2}
\end{array}\right]>0, \quad i=1, \ldots, n_{u} .
$$

The appropriate condition on the derivative of the function $x^{T} P x$ is guaranteed by the fact that condition

$$
q^{T} W(H x+G w+K x+L q-q) \geq 0,(x, q, w) \neq 0
$$

implies

$$
x^{T}\left(A^{T} P+P A\right) x+2 x^{T} P(B q+E w)<-\frac{1}{\gamma^{2}} z^{T} z+w^{T} w,
$$

which generalizes the inequalities (7) and (33) addressing the same problem, respectively for the case without saturation and for the case with a global sector bound on the saturation. As compared to (7) and (33) the right hand side of the inequality above is divided by $\gamma$. This is a key fact which allows to write inequality (49) independently of $\gamma$ and therefore to derive the LMIs (54) below for the regional $\mathcal{L}_{2}$ gain computation.

Using the definition of $z$, and applying the S-procedure and Schur complements, produces the matrix conditions

$$
\begin{aligned}
& 0>\left[\begin{array}{cccc}
A^{T} P+P A & P B+K^{T} W+H^{T} W & P E & C^{T} \\
\star & W L+L^{T} W-2 W & W G & D^{T} \\
\star & \star & -I & F^{T} \\
\star & \star & \star & -\gamma^{2} I
\end{array}\right] \\
& =\operatorname{He}\left[\begin{array}{cccc}
P A & P B & P E & 0 \\
W H+W K & W L-W & W G & 0 \\
0 & 0 & -I / 2 & 0 \\
C & D & F & -\gamma^{2} I / 2
\end{array}\right] \text {. }
\end{aligned}
$$


The four blocks in the upper left corner correspond the the LMI for internal stability given in (44). Like before, the matrix conditions (51) and (53) are not linear in the free variables $P, W$, and $H$. Again, notice the $H^{T} W$ term that appears but that the matrix conditions can be reformulated as LMIs through a nonlinear transformation on the free variables. Using the same transformation as in the case for internal stability, the following LMIs are obtained in the free variables $Q, U$ and $Y$ :

$$
\begin{aligned}
& Q>0 \\
& U> \\
& \\
& 0>\operatorname{He}\left[\begin{array}{cccc}
A Q & B U & E & 0 \\
Y+K Q & L U-U & G & 0 \\
0 & 0 & -I / 2 & 0 \\
C Q & D U & F & -\gamma^{2} I / 2
\end{array}\right] \\
& {\left[\begin{array}{cc}
Q & Y_{i}^{T} \\
Y_{i} & \bar{u}_{i}^{2} / s^{2}
\end{array}\right]>0, \quad i=1, \ldots, n_{u} . }
\end{aligned}
$$

The minimum $\gamma^{2}>0$ for which the LMIs are feasible is a nondecreasing function of $s$. An upper bound on the nonlinear gain from $\|w\|_{2}$ to $\|z\|_{2}$ can be established by solving the LMI eigenvalue problem of minimizing $\gamma^{2}$ subject to the LMIs (54) for a wide range of values for $s$ and plotting $\gamma$ as a function of $s$.

Next, an example of nonlinear gain computation is provided to illustrate the use of the LMIs (54).

Example 4 Consider a one-dimensional plant stabilized by negative feedback through a unit saturation and subject to a scalar disturbance $w$. The closed-loop dynamic equation is given by

$$
\begin{aligned}
\dot{x}_{p} & =a x_{p}+\operatorname{sat}(u)+w \\
u & =-(a+10) x_{p},
\end{aligned}
$$

which can be written in terms of the deadzone function as

$$
\begin{aligned}
\dot{x}_{p} & =-10 x_{p}+q+w \\
q & =\mathrm{dz}\left((a+10) x_{p}\right) .
\end{aligned}
$$

System (55) will be analyzed in three main cases:

1. $a=1$, which resembles an exponentially unstable plant stabilized through a saturated loop;

2. $a=0$, which represents an integrator, namely a marginally stable plant stabilized through a saturated loop;

3. $a=-1$, which represents an exponentially stable plant whose speed of convergence is increased through a saturated loop.

When adopting the representation (3), system (55) is described by the following selection, with $z=x_{p}$ :

$$
\left[\begin{array}{c|c|c}
A & B & E \\
\hline C & D & F \\
\hline K & L & G
\end{array}\right]=\left[\begin{array}{c|c|c}
-10 & -1 & 1 \\
\hline 1 & 0 & 0 \\
\hline-a-10 & 0 & 0
\end{array}\right],
$$

The matrices (56) can be used in the LMI conditions (54) for different values of $s$, to compute the nonlinear gains for the three cases under consideration. The resulting curves are shown in Figure 5 where it is possible to appreciate the three different behaviors characterizing exponentially stable plants, non exponentially unstable plants with poles on the imaginary axis and exponentially unstable plants.

In particular, for exponentially stable plants the global $\mathcal{L}_{2}$ gain is finite so that the nonlinear $\mathcal{L}_{2}$ approaches the finite $\mathcal{L}_{2}$ gain of the uncontrolled plant as $s$ grows arbitrarily large. For non exponentially stable plants there isn't a global finite $\mathcal{L}_{2}$ gain so that the curve admits a finite value for each $s$ but grows arbitrarily large for increasing values of $s$. Finally, for exponentially unstable plants, there is a large enough value of $s$ for which the gain becomes unbounded, so that there is a finite value of $s$ such that the nonlinear $\mathcal{L}_{2}$ gain grows unbounded approaching that value. 


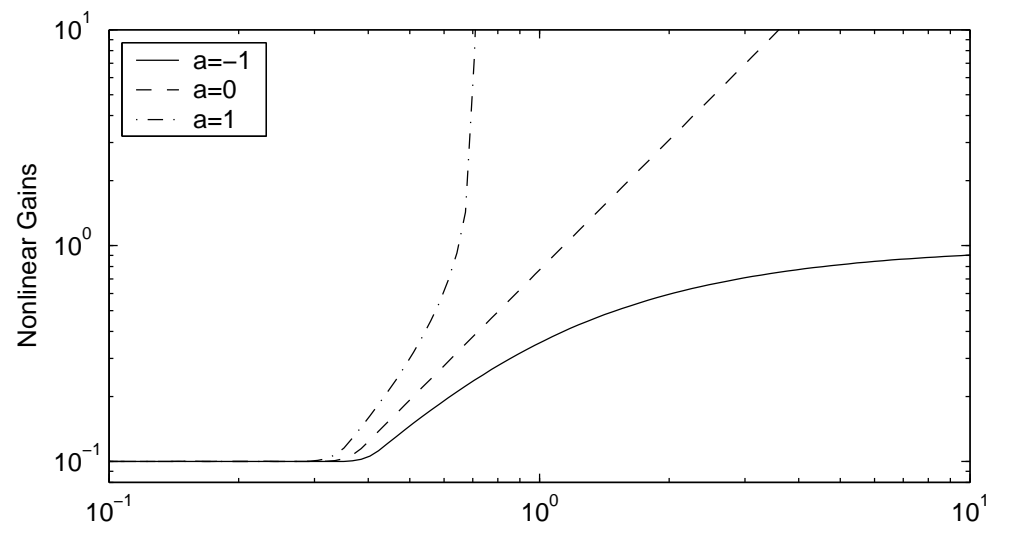

Figure 5: Nonlinear $\mathcal{L}_{2}$ gains for the three cases considered in Example 4.

\section{Regional synthesis for external stability}

This section addresses synthesis in feedback loops with saturation based on the LMIs that were derived earlier in this chapter. Only the case of regional $\mathcal{L}_{2}$ external stability is described. The purpose of this chapter is to give an indication of the types of calculations that arise in the synthesis of anti-windup algorithms.

\subsection{LMI formulations of anti-windup synthesis}

In typical anti-windup synthesis, the designer can inject the deadzone nonlinearity, driven by the control input, at various places in the feedback loop and can also inject the state of a filter driven by the deadzone nonlinearity. Letting $x$ denote the composite state of the plant having $n_{p}$ components, unconstrained controller having $n_{c}$ components, and anti-windup filter having $n_{a w}$ components, the synthesis problem can be written as

$$
\begin{aligned}
\dot{x} & =\bar{A} x+\bar{B}_{0}(u-q)+\bar{B}_{1}\left(K_{1} \bar{C}_{1} x+\Phi_{1} q\right)+\bar{E} w \\
z & =\bar{C}_{2} x+\bar{D} q+\bar{F} w \\
u & =\bar{K}_{0} x+\bar{L} q+K_{2} \bar{C}_{1} x+\Phi_{2} q+\bar{G} w \\
q & =u-\operatorname{sat}(u)
\end{aligned}
$$

where the design parameters are $K_{1}, K_{2}, \Phi_{1}$ and $\Phi_{2}$. All of the other matrices are fixed by the problem description, coming either from the plant model or the model of the unconstrained controller and are overlined for notational convenience. Typically $\bar{C}_{1} x$ represents the states of the anti-windup augmentation filter. The design parameters $K_{1}$ and $K_{2}$ determine how those states are used to determine the characteristics of the filter and the injected terms in the controller. When using static anti-windup augmentation, which corresponds to the case where there are no states in the anti-windup augmentation filter, the matrices $K_{1}$ and $K_{2}$ are set to zero. The design parameters $\Phi_{1}$ and $\Phi_{2}$ determine how the deadzone nonlinearity is injected into the state equations and controller. The matrix $\bar{B}_{1}$ limits where the states of the anti-windup filter and the deadzone nonlinearity can be injected into the dynamical equations.

With the definitions

$$
\begin{aligned}
& {\left[\begin{array}{c|c|c}
A & B & E \\
\hline C & D & F \\
\hline K & L & G
\end{array}\right]=} \\
& {\left[\begin{array}{c|c|c}
\bar{A}+\bar{B}_{0} \bar{K}_{0}+\left(\bar{B}_{1} K_{1}+\bar{B}_{0} K_{2}\right) \bar{C}_{1} & \bar{B}_{0}\left(\bar{L}+\Phi_{2}-I\right)+\bar{B}_{1} \Phi_{1} & \bar{E}+\bar{B}_{0} \bar{G} \\
\hline \bar{C}_{2} & \bar{D} & \bar{F} \\
\hline & \bar{K} & \bar{G}+\Phi_{2}
\end{array}\right],}
\end{aligned}
$$

the system (57) agrees with the system (3) and the corresponding regional performance analysis LMI from 
Section 5.3 is

$$
\begin{aligned}
Q & >0 \\
U & >0 \text { diagonal } \\
0 & >\left[\begin{array}{cccc}
Q A^{T}+A Q & B U+Q K^{T}+Y^{T} & E & Q C^{T} \\
\star & L U+U L^{T}-2 U & G & U D^{T} \\
\star & \star & -I & F^{T} \\
\star & \star & \star & -\gamma^{2} I
\end{array}\right] \\
{\left[\begin{array}{cc}
Q & Y_{i}^{T} \\
Y_{i} & \bar{u}_{i}^{2} / s^{2}
\end{array}\right] } & >0, i=1, \ldots, n_{u} .
\end{aligned}
$$

Since some of the components of $A, B, K$, and $L$ are free for design, these matrices are replaced in (59) by their definitions from (58). The additional definitions

$$
\begin{aligned}
& \Theta_{1}:=\Phi_{1} U \\
& \Theta_{2}:=\Phi_{2} U
\end{aligned}
$$

give relationships between $\Phi_{i}$ and $\Theta_{i}$ for $i \in\{1,2\}$ that are invertible because $U$ is positive definite by assumption.

Using these definitions, the matrix conditions (59) become

$$
\begin{aligned}
Q & >0 \\
U & >0 \text { diagonal } \\
0 & >\operatorname{He}\left[\begin{array}{cccc}
\left(\bar{A}+\bar{B}_{0} \bar{K}_{0}\right) Q+\left(\bar{B}_{1} K_{1}+\bar{B}_{0} K_{2}\right) \bar{C}_{1} Q & \bar{B}_{1} \Theta_{1}+\bar{B}_{0}\left((\bar{L}-I) U+\Theta_{2}\right) & \bar{B}_{0} \bar{G}+\bar{E} & Q \bar{C}_{2}^{T} \\
\bar{K}_{0} Q+K_{2} \bar{C}_{1} Q+Y & \Theta_{2}+(\bar{L}-I) U & \bar{G} & U \bar{D}^{T} \\
0 & 0 & -\frac{1}{2} I & \bar{F}^{T} \\
0 & 0 & -\frac{1}{2} \gamma^{2} I
\end{array}\right] \\
0 & >-\left[\begin{array}{cc}
Q & Y_{i}^{T} \\
Y_{i} & \bar{u}_{i}^{2} / s^{2}
\end{array}\right], i=1, \ldots, n_{u} .
\end{aligned}
$$

In the case of static anti-windup augmentation, where $K_{1}=0$ and $K_{2}=0$, the conditions (61) constitute LMIs in the free variables $U, Q, \Theta_{1}, \Theta_{2}$, and $\gamma$. Note that in (61) all the fixed parameters are overlined and all the free variables are not.

For some problems related to anti-windup synthesis, $\bar{C}_{1}$ is invertible and then, with the definitions

$$
\begin{aligned}
& X_{1}:=K_{1} \bar{C}_{1} Q \\
& X_{2}:=K_{2} \bar{C}_{1} Q
\end{aligned},
$$

which give an invertible relationship between $K_{i}$ and $X_{i}, i \in\{1,2\}$, the conditions (61) constitute LMIs in the free variables $U, Q, \Theta_{1}, \Theta_{2}, X_{1}, X_{2}$, and $\gamma$.

In the more typical situation where $\bar{C}_{1}$ is not invertible, a different approach can be taken to eliminate the nonlinear terms that involve products of $Q$ and $K_{i}, i \in\{1,2\}$, at least when the size of the anti-windup augmentation filter has the same number of states as the plant model. In this situation, define

$$
\Lambda:=\left[\begin{array}{cc}
K_{1} & \Theta_{1} \\
K_{2} & \Theta_{2}
\end{array}\right]
$$

and construct matrices $\Psi, H$ and $G$ such that the conditions (61) become

$$
\begin{aligned}
Q & >0 \\
U & >0 \\
0 & >\operatorname{He}(\Psi+H \Lambda G) \\
0 & >\left[\begin{array}{cc}
Q & Y_{i}^{T} \\
Y_{i} & \bar{u}_{i}^{2} / s^{2}
\end{array}\right], i=1, \ldots, n_{u} .
\end{aligned}
$$


According to the "Elimination lemma" from linear algebra, there exists a value $\Lambda$ satisfying $0>$ $\mathrm{He}(\Psi+H \Lambda G)$ if and only if

$$
\begin{aligned}
& 0>W_{H}^{T} \Psi W_{H} \\
& 0>W_{G}^{T} \Psi W_{G}
\end{aligned}
$$

where $W_{H}$ and $W_{G}$ are any full rank matrices satisfying $W_{H}^{T} H=0$ and $G W_{G}=0$.

Then, exploiting the special structure of anti-windup problems, the matrices $Q$ and $Y$ can be partitioned as

$$
Q=\left[\begin{array}{cc}
{\left[\begin{array}{cc}
R_{11} & R_{12} \\
R_{12}^{T} & R_{22}
\end{array}\right]} & N \\
N^{T} & M
\end{array}\right], P=Q^{-1}=\left[\begin{array}{cc}
S^{-1} & P_{12} \\
P_{12}^{T} & P_{22}
\end{array}\right], Y=\left[\begin{array}{lll}
Z & Y_{b} & Y_{c}
\end{array}\right]
$$

where $R_{11}$ is an $n_{p} \times n_{p}$ matrix, $R_{22}$ is an $n_{c} \times n_{c}$ matrix, and $M$ in an $n_{a w} \times n_{a w}$ matrix, and it can be verified that $W_{H}^{T} \Psi W_{H}<0$ is an LMI in $R_{11}$ and $Z$, while $W_{G}^{T} \Psi W_{G}<0$ is an LMI in $S$. Then, as long as $R_{11}$ and $S$ satisfy the condition $R_{11}-S_{11}>0$, which is another LMI in $R_{11}$ and $S$, it is always possible to pick $R_{12}, R_{22}, N$, and $M$ so that $P Q=I$. Finally, as long as

$$
0<\left[\begin{array}{cc}
R_{11} & Z_{i}^{T} \\
Z_{i} & \bar{u}_{i}^{2} / s^{2}
\end{array}\right], i=1, \ldots, n_{u},
$$

which is yet another LMI in $R_{11}$ and $Z$, it is always possible to pick $Y_{b}$ and $Y_{c}$ so that

$$
0<\left[\begin{array}{cc}
Q & Y_{i}^{T} \\
Y_{i} & \bar{u}_{i}^{2} / s^{2}
\end{array}\right], i=1, \ldots, n_{u} .
$$

Finally, with $Y$ and $Q$ generated, it is possible to find $\Lambda$ and $U$ such that $0>\operatorname{He}(\Psi+H \Lambda G)$.

\subsection{Restricting the size of matrices}

It is sometimes convenient to restrict the size of the matrices determined by the LMI solver when seeking for optimal solutions minimizing the gain $\gamma^{2}$ in (61). Indeed, it may sometimes happen that minimizing the gain leads the LMI solver in a direction where certain parameters become extremely large providing very little performance increase. To avoid this undesirable behavior, it is often convenient to restrict the size of the free variables in (61) by incorporating extra constraints in the LMI optimization.

Given any free matrix variable $M$, one way to restrict its entries is to impose a bound $\kappa$ on its maximum singular value, namely impose

$$
M^{T} M<\kappa^{2} I .
$$

This can be done by dividing the equation above by $\kappa$ and applying a Schur complement to $\kappa I-M^{T} \frac{1}{\kappa} M>0$, which gives:

$$
\left[\begin{array}{cc}
\kappa I & M^{T} \\
M & \kappa I
\end{array}\right]>0
$$

This type of solution can be used, for example, to restrict the size of the anti-windup matrices $K_{1}, K_{2}, \Phi_{1}$ and $\Phi_{2}$ in (61). In particular, this is achieved by imposing

$$
\left[\begin{array}{cc}
\kappa I & \Lambda^{T} \\
\Lambda & \kappa I
\end{array}\right]>0, \quad U>I
$$

where $\Lambda$ is defined in equation (63). Then, since by (60)

$$
\left[\begin{array}{ll}
K_{1} & \Phi_{1} \\
K_{2} & \Phi_{2}
\end{array}\right]=\left[\begin{array}{ll}
K_{1} & \Theta_{1} \\
K_{2} & \Theta_{2}
\end{array}\right]\left[\begin{array}{cc}
I & 0 \\
0 & U^{-1}
\end{array}\right]
$$

the anti-windup parameters will satisfy $\left|\left[\begin{array}{cc}K_{1} & \Phi_{1} \\ K_{2} & \Phi_{2}\end{array}\right]\right| \leq|\Lambda|\left|U^{-1}\right| \leq|\Lambda| \leq \kappa$, where $|\cdot|$ denotes the maximum singular value of its argument.

Notice that imposing the extra constraints (66) reduces the feasibility set of the synthesis LMIs and conservatively enforces the bound on the anti-windup matrices (because it also restricts the set of allowable free variables $U)$. However it works well in many practical cases. 


\section{$7 \quad$ Notes and references}

LMIs have played a foundational role in analysis and control of dynamical systems for several decades. A comprehensive book on this topic is the classic [1], where an extensive list of references can be found. That book also contains the facts quoted herein concerning Schur complements, Finsler Lemma, the S-procedure, and the elimination lemma. The analysis of Section 4 corresponds to the MIMO version of the classical circle criterion in state-space form. The regional analysis of Section 5 is primarily due to the generalized sector condition introduced in [15] and [3].

The global and regional analysis discussed in Sections 4 and 5 corresponds to the quadratic results of [16] where also nonquadratic stability and $\mathcal{L}_{2}$ performance estimates are given. The synthesis method of Section 6 corresponds to the regional techniques of [17] for the most general case but previous papers followed that approach for anti-windup synthesis: the static global design of [23], the dynamic global design of [12, 26] and its external extension in [14], the discrete-time results of [24] and [21]. The "Elimination Lemma" used in Section 6 can be found in [1]. It was first used as shown here in the context of LMI-based $\mathcal{H}_{\infty}$ controller synthesis. The corresponding techniques appeared simultaneously and independently in [7] and [19] (see also, [6] for an explicit solution to the second design step when applying the elimination lemma).

Well posedness for feedback loops involving saturation nonlinearities has been first addressed in [27], where results from [2] were used to establish sufficient conditions for well posedness. Similar tools were also used in [12], where the well posedness of the earlier schemes of [23] was also proved. The strong well posedness constraint discussed in Section 4.2 arises from the results of [13]. More recently, in [16] a further characterization of sufficient only and necessary and sufficient conditions has been given.

Regarding the LMI solvers mentioned in Examples 1-3, the LMI control toolbox [8] of MATLAB can be purchased together with MATLAB. YALMIP [20] is a modeling language for defining and solving advanced optimization problems. CVX [11] is a package for specifying and solving convex programs. Both [20] and CVX [11] are extensions of MATLAB which can be downloaded from the web for free and easily installed as toolboxes on a MATLAB installation.

\section{References}

[1] S. Boyd, L. El Ghaoui, E. Feron, and V. Balakrishnan. Linear Matrix Inequalities in System and Control Theory. Society for Industrial an Applied Mathematics, 1994.

[2] F.H. Clarke. Optimization and Nonsmooth Analysis. Society for Industrial an Applied Mathematics, 1990.

[3] J.M. Gomes da Silva Jr and S. Tarbouriech. Anti-windup design with guaranteed regions of stability: an LMI-based approach. IEEE Trans. Aut. Cont., 50(1):106-111, 2005.

[4] D. Dai, T. Hu, A.R. Teel, and L. Zaccarian. Control of saturated linear plants via output feedback containing an internal deadzone loop. In American Control Conference, pages 5239-5244, Minneapolis (MN), USA, June 2006.

[5] D. Dai, T. Hu, A.R. Teel, and L. Zaccarian. Piecewise-quadratic Lyapunov functions for systems with deadzones or saturations. Systems and Control Letters, 58:365-371, 2009.

[6] P. Gahinet. Explicit controller formulas for LMI-based $\mathcal{H}_{\infty}$ synthesis. Automatica, 32(7):1007-1014, July 1996.

[7] P. Gahinet and P. Apkarian. A linear matrix inequality approach to $\mathcal{H}_{\infty}$ control. Int. J. Robust and Nonlinear Control, 4:421-448, 1994.

[8] P. Gahinet, A. Nemirovski, A.J. Laub, and M. Chilali. LMI Control Toolbox. The MathWorks Inc., 1995. 
[9] S. Galeani, M. Massimetti, A.R. Teel, and L. Zaccarian. Reduced order linear anti-windup augmentation for stable linear systems. International Journal of Systems Science, 37(2):115-127, 2006.

[10] S. Galeani, A.R. Teel, and L. Zaccarian. Constructive nonlinear anti-windup design for exponentially unstable linear plants. Systems and Control Letters, 56(5):357-365, 2007.

[11] M. Grant and S. Boyd. CVX: Matlab software for disciplined convex programming. (web page and software). http://stanford. edu/ boyd/cvx.

[12] G. Grimm, J. Hatfield, I. Postlethwaite, A.R. Teel, M.C. Turner, and L. Zaccarian. Antiwindup for stable linear systems with input saturation: an LMI-based synthesis. IEEE Trans. Aut. Cont. (A), 48(9):1509-1525, September 2003.

[13] G. Grimm, A.R. Teel, and L. Zaccarian. Establishing Lipschitz properties of multivariable algebraic loops with incremental sector nonlinearities. In Conference on Decision and Control, Maui (HI), USA, December 2003.

[14] G. Grimm, A.R. Teel, and L. Zaccarian. Linear LMI-based external anti-windup augmentation for stable linear systems. Automatica (B), 40(11):1987-1996, 2004.

[15] T. Hu, Z. Lin, and B.M. Chen. An analysis and design method for linear systems subject to actuator saturation and disturbance. Automatica, 38(2):351-359, 2002.

[16] T. Hu, A.R. Teel, and L. Zaccarian. Stability and performance for saturated systems via quadratic and non-quadratic Lyapunov functions. IEEE Trans. Aut. Cont. (A), 51(11):1770-1786, November 2006.

[17] T. Hu, A.R. Teel, and L. Zaccarian. Anti-windup synthesis for linear control systems with input saturation: achieving regional, nonlinear performance. Automatica (B), 44(2):512-519, 2008.

[18] T. Hu, A.R. Teel, and L. Zaccarian. Stability and performance for saturated systems via quadratic and non-quadratic Lyapunov functions. Technical report, RR-06.55, Dipart. di Inf., Sist. e Prod., University of Rome, Tor Vergata, March 2006.

[19] T. Iwasaki and R.E. Skelton. All controllers for the general $\mathcal{H}_{\infty}$ control problem: LMI existence conditions and state space formulas. Automatica, 30(8):1307-17, August 1994.

[20] J. Lofberg. YALMIP : A toolbox for modeling and optimization in MATLAB. In Proceedings of the CACSD Conference, Taipei, Taiwan, 2004.

[21] M. Massimetti, L. Zaccarian, T. Hu, and A.R. Teel. LMI-based linear anti-windup for discrete time linear control systems. In Conference on Decision and Control, pages 6173-6178, San Diego (CA), USA, December 2006.

[22] M. Massimetti, L. Zaccarian, T. Hu, and A.R. Teel. Linear discrete-time global and regional antiwindup: an LMI approach. Int. Journal of Control, 2009, to appear.

[23] E.F. Mulder, M.V. Kothare, and M. Morari. Multivariable anti-windup controller synthesis using linear matrix inequalities. Automatica, 37(9):1407-1416, September 2001.

[24] A. Syaichu-Rohman and R.H. Middleton. Anti-windup schemes for discrete time systems: an lmi-based design. In Asian Control Conference, pages 554-561, Melbourne (VIC), Australia, July 2004.

[25] S. Tarbouriech and M. Turner. Anti-windup design: an overview of some recent advances and open problems. Control Theory \& Applications, IET, 3(1):1-19, 2009.

[26] F. Wu and B. Lu. Anti-windup control design for exponentially unstable LTI systems with actuator saturation. Systems and Control Letters, 52(3-4):304-322, 2004.

[27] L. Zaccarian and A.R. Teel. A common framework for anti-windup, bumpless transfer and reliable designs. Automatica (B), 38(10):1735-1744, 2002. 